\title{
Le rôle de l'Etat dans la construction des chemins de fer du Portugal au XIXe siècle
}

\section{Magda de Avelar Pinheiro}

\section{Résumé}

Résumé Le retard économique a obligé l'Etat portugais - dont la situation financière était caractérisée par l'insuffisance des recettes fiscales - à assurer la construction des chemins de fer les moins rentables et à aider la construction des autres. Bien que le rôle de ses ingénieurs ait été important, l'effort financier n'a pas assuré à l'Etat un contrôle absolu des tracés et des tarifs ferroviaires. Les régions intérieures en ont souffert. Le Portugal n'a pas dépassé le seuil du développement pendant cette période mais il a connu une croissance considérable.

\begin{abstract}
Abstract Economie backwardness forced Portuguese State, wich didn't collect enough taxes in the 19th century, to provide financial assistance to railway building. Although Portuguese engineers have been very importante, financial effort didn't give the State complete control on railway lines and maximum rates. Hinterland was handicapped. Portugal knew a remarkable progress within this period although it didn't reach take off.
\end{abstract}

\section{Citer ce document / Cite this document :}

de Avelar Pinheiro Magda. Le rôle de l'Etat dans la construction des chemins de fer du Portugal au XIXe siècle. In: Histoire, économie et société, $1992,11^{\mathrm{e}}$ année, $\mathrm{n}^{\circ} 1$. Les transports terrestres en Europe Continentale (XIXe-XXe siècles) pp. 173-184; doi : 10.3406/hes.1992.1630

http://www.persee.fr/doc/hes_0752-5702_1992_num_11_1_1630

Document généré le 15/06/2016 


\title{
LE ROLE DE L'ETAT DANS LA CONSTRUCTION DES CHEMINS DE FER DU PORTUGAL AU XIXe SIECLE
}

\author{
par Magda DE AVELAR PINHEIRO
}

\section{Résumé}

Le retard économique a obligé l'Etat portugais - dont la situation financière était caractérisée par l'insuffisance des recettes fiscales - à assurer la construction des chemins de fer les moins rentables et à aider la construction des autres.

Bien que le rôle de ses ingénieurs ait été important, l'effort financier n'a pas assuré à l'Etat un contrôle absolu des tracés et des tarifs ferroviaires. Les régions intérieures en ont souffert.

Le Portugal n'a pas dépassé le seuil du développement pendant cette période mais il a connu une Itroissance considérable.

Abstract

Economic backwardness forced portuguese State, wich didn't collect enough taxes in the 19th century, to provide financial assistance to railway building.

Although portuguese engineers have been very importante, financial effort didn't give the State complete control on railway lines and maximum rates. Hinterland was handicapped.

Portugal knew a remarkable progress within this period although it didn't reach take off.

La construction des chemins de fer dans le Portugal du XIXe siècle s'est, pour l'essentiel, déroulée entre 1853 et 1891 . Après cette date, seules les lignes déjà en voie de construction ont été terminées. Les débuts furent tardifs, trente et une années s'étant écoulées entre l'inauguration du premier tronçon de chemin de fer en Angleterre et au Portugal.

Ce retard n'est pas le résultat de l'indifférence et il fut vécu avec impatience par une «intelligentsia» qui avait connu l'exil en France, en Belgique ou en Angleterre. Les chemins de fer étaient, cependant, très chers : pour les construire avec des capitaux privés nationaux, il aurait fallu pouvoir compter sur des structures de crédit capables de mobiliser des capitaux, drainant des épargnes jusque là improductives.

L'évolution du Portugal depuis la première décennie du XIXe siècle n'avait pas été particulièrement favorable au développement des structures de crédit. Dans les années 1820 , la perte progressive du commerce brésilien a privé la bourgeoisie 
portugaise d'une importante source d'accumulation de capitaux tandis qu'après 1834 la vente des biens nationaux a absorbé une partie des disponibilités!.

Les capitalistes étrangers ne se sont pas montrés particulièrement empressés d'investir dans les chemins de fer d'un pays qui ne réussissait pas à assurer le paiement de ses dépenses publiques et qui, depuis 1837 , avait souvent oublié les échéances de paiement des intérêts de sa dette extérieure.

L'appui direct de l'Etat ou la garantie d'un taux d'intérêt minimum aux capitaux investis ont toujours été exigés et c'est seulement après la fin de la période révolutionnaire, le 18 décembre 1852 , que la conversion forcée de la dette en $3 \%$ perpétuel a permis le paiement régulier des intérêts de la dette publique, rendant ainsi crédible la négociation de contrats incluant ce type de charges. La période de construction des chemins de fer se termine aussi par une crise des finances de l'État marquée par une nouvelle impossibilité d’assurer le paiement des charges de la dette publique.

Les investissements de l'Etat dans les chemins de fer étaient destinés à compenser des risques que les capitaux privés estimaient, à juste titre d'ailleurs, trop importants. Cette exigence d'une garantie d'intérêt représentait la conscience d'un retard économique qui ne permettait pas aux compagnies privées d'obtenir des capitaux à des taux suffisamment faibles ou d'atteindre des recettes d'exploitation suffisamment élevées pour assurer les charges des investissements. Pour l'Etat portugais qui ne réussissait pas à équilibrer les dépenses ordinaires avec les recettes fiscales, il s'agissait de retirer des taux d'intérêt peu élevés ou nuls sur des capitaux obtenus à des taux d'intérêt très élevés ${ }^{2}$.

En investissant de cette façon, l'Etat s'efforçait d'aider la rentabilisation de capitaux privés investis dans d'autres secteurs, comme l'agriculture, l'industrie ou le commerce. L'objectif était d'accélérer la croissance économique ou, en utilisant les mots de l'époque, le progrès. L'Etat trichait ainsi par rapport à l'utopie de l'équidistance libérale, mais la justification de son attitude résidait dans la croyance que la construction des chemins de fer, en contribuant à égaliser les conditions de transport dans le pays, remettait en action les mécanismes auto-régulateurs du marché ${ }^{3}$. Pour justifier cette action que d'autres Etats avaient entreprise plus tôt, loriginalité n'était pas nécessaire.

Dès 1851 , la construction des chemins de fer par l'Etat conformément à l'exemple belge était considérée comme souhaitable mais aussi peu réalisable, étant donné les difficultés rencontrées pour augmenter les impôts et la rigidité d'une partie des dépenses ${ }^{4}$.

Malgré cela, l'Etat a dépensé 60871 millions de reis pendant 39 ans à une moyenne annuelle de 1500 millions de reis (8 400000 francs ou 330 mille livres au pair) pour aider à la construction et au fonctionnement des chemins de fer. Ces dépenses représentent $29,5 \%$ du déficit cumulé des dépenses publiques, que nous avons évalué pour cette période (tableau 2). Il faut cependant en déduire les recettes 
des chemins de fer de l'Etat et l'impôt sur les transports ferroviaires, ce qui ramène le total à $\mathbf{4 8} 800$ millions de reis 5 .

Les modalités de l'appui de l'Etat ayant évolué dans le temps, ces dépenses correspondent soit à la construction et à l'exploitation des chemins de fer de l'Etat, soit à des subventions et à des garanties d'intérêt aux compagnies privées, soit encore au rachat de chemins de fer déjà construits et à la fiscalisation des constructions.

Les effets des divers types de dépenses sur le développement économique du pays et sur les finances de l'Etat étaient différents. Par rapport au déficit des finances, le pourcentage le plus fort se situe entre 1871-72 et 1880-81 à une époque où l'Etat a construit directement. Cependant, les pourcentages les plus élevés des dépenses ferroviaires se situent en 1861-62 et en 1862-63 quand elles atteignent presque 30\% des recettes fiscales (tableau 1). Il s'agit pour ces années-là de subventions payées aux compagnies privées sous forme de titres de la dette publique consolidée à la cotation du jour. Ce mode de paiement eut comme conséquence une croissance rapide de la dette consolidée dont les charges devinrent de 1866 à 1870 presque impossibles à supporter.

Les pourcentages relativement faibles des années 1880 doivent être corrigés car des paiements ont été effectués par le biais de la dette flottante que nous ne pouvons pas estimer. Ces pourcentages peu élevés sont aussi dus au système de garanties d'intérêt dont les effets se sont fait sentir plus tard.

L'Etat a distribué 15850,3 millions de reis de subventions de construction aux compagnies privées pendant toute la période, ce qui correspond à $26 \%$ de toutes ses dépenses dans ce secteur.

Les garanties d'intérêt qui ont été payées jusqu'en 1891 n'atteignirent que 440,3 millions de reis, mais leur montant cumulé s'élevait en 1911 à 8432,495 millions de reis ${ }^{6}$.

En 1879, l'Etat détenait $50 \%$ des voies de chemin de fer construites dans le pays. Ce résultat ne provenait pas d'une politique déterminée ayant cet objectif mais plutôt des avatars de la vie des compagnies privées confrontées à des recettes d'exploitation insuffisantes.

Dès 1853 , l'Etat a été obligé d'acheter $1 / 3$ des actions de la «Central Peninsulan» pour rendre viable la constitution de l'entreprise. Il a dépensé 1059,9 millions de reis dans cette opération ${ }^{7}$. Le rachat de la totalité des actions a été nécessaire pour résoudre la crise de l'entreprise et, en 1859-60, le chemin de fer avait déjà coûté 4460,6 millions de reis à l'Etat ; il fut alors vendu à D. José de Salamanca par 2700 millions de reis. L'Etat a aussi acheté, à la même époque, le chemin de fer du Sud pour éviter une vente à Salamanca, ce qui aurait donné à ce dernier le monopole de l'accès à la capitale. Ces chemins de fer ont été revendus et leur rachat n'avait pour objectif que la solution à la crise et la constitution de nouvelles entreprises. 


\section{Tableau 1}

Dépenses ferroviaires de l'Etat en pourcentage des recettes

\begin{tabular}{|c|c|c|c|c|c|}
\hline années & $\%$ & années & $\%$ & années & $\%$ \\
\hline $1853-54$ & 3,8 & $1865-66$ & 2,6 & $1877-78$ & 15,6 \\
\hline $1854-55$ & 3,3 & $1866-67$ & 0,4 & $1878-79$ & 7,8 \\
\hline $1855-56$ & 6,2 & $1867-68$ & 0,3 & $1879-80$ & 7,4 \\
\hline $1856-57$ & 7,8 & $1868-69$ & 0,5 & $1880-81$ & 12,5 \\
\hline $1857-58$ & 15,8 & $1869-70$ & 16,2 & $1881-82$ & 8,9 \\
\hline $1858-59$ & 5,4 & $1870-71$ & 1,3 & $1882-83$ & 3,4 \\
\hline $1859-60$ & 3,3 & $1871-72$ & 1,2 & $1883-84$ & 7,0 \\
\hline $1860-61$ & 3,1 & $1872-73$ & 3,4 & $1884-85$ & 6,9 \\
\hline $1861-62$ & 28,8 & $1873-74$ & 7,5 & $1885-86$ & 6,8 \\
\hline $1862-63$ & 29,7 & $1874-75$ & 10,3 & $1886-87$ & 8,1 \\
\hline $1863-64$ & 11,6 & $1875-76$ & 12,0 & $1887-88$ & 3,1 \\
\hline $1864-65$ & 4,9 & $1876-77$ & 14,3 & $1888-89$ & 4,1 \\
\hline & & & & $1889-90$ & 4,4 \\
\hline
\end{tabular}

Tableau établi d'après les rapports des ministres des Finances et des Travaux Publics et les Comptes Généraux de l'Etat.

Tableau 2

Déficits et dépenses ferroviaires de l'Etat en milliers de Reis

\begin{tabular}{|l|r|r|c|}
\hline Années & Déficit cumulé & Dépenses cumulées & $\%$ \\
\hline $1852-53$ & 29250997 & 5735057 & 19,6 \\
$1860-61$ & & & \\
$1861-62$ & 46694261 & 14737462 & 31,6 \\
$1870-71$ & 56002836 & 23117332 & 41,3 \\
$1871-72$ & 74484885 & 17161377 & 23,1 \\
$1881-82$ & & & \\
\hline
\end{tabular}


La crise de 1866, dont la gravité politique et sociale fut moindre qu'en Espagne, a durement touché les compagnies ferroviaires et elle a obligé l'Etat à racheter la compagnie du Sud Est. Liée à la dette flottante du pays, la faillite de la compagnie lors du krach du 11 mai 1866 rendait impossible la levée des emprunts nécessaires au paiement des charges de la dette consolidée.

Cette crise a cependant fait naître un courant nationaliste prônant la construction et l'administration des chemins de fer par l'Etat. Le chemin de fer de «Douro e Minho» fut initialement construit par l'Etat et le chemin de fer du Sud et Sud Est est demeuré sa propriété jusqu'en 1926. Vers 1884 il n'était pas nécessaire d'être socialiste pour plaider le rachat des lignes de la Compagnie Royale 8 . De 1874 à 1877 , l'Etat a émis sur le marché intérieur des obligations $6 \%$ amortissables. Leur taux d'intérêt effectif moyen s'est élevé à 6,63\%. A cette date, il était devenu moins difficile de lever des capitaux dans le pays ; les Portugais avaient amorcé leur percée dans le conseil d'administration de Compagnie Royale des Chemins de Fer et l'expansion des institutions bancaires était notoire. Les Portugais préféraient, cependant, acheter des fonds publics qu'ils estimaient plus sûrs.

Jusqu'en 1891 l'Etat a destiné $38,6 \%$ du total de ses dépenses ferroviaires aux chemins de fer qu'il administrait directement. Il a aussi consacré $6 \%$ de ses dépenses au rachat des chemins de fer du Sud (938,73 millions de reis) et du Sud Est $(2705,6$ millions de reis). A la même époque, il détenait $38 \%$ du réseau. Ce réseau de l'État qui était né par suite des faibles rendements des compagnies privées ou qui avait été construit là où personne ne voulait le construire, n'a jamais pu dépasser le seuil de rentabilité. De 1880 à 1890 , les recettes de l'exploitation des chemins de fer du Sud et du Sud Est n'ont pas dépassé en moyenne 2,6\% du capital investi9.

L'Etat a conservé, en théorie, le droit de décision sur le tracé du réseau ferroviaire. Sauf dans le cas du chemin de fer du Sud jusqu'à Vendas, toutes les études ayant servi de base aux concours ont été faites pour son compte. Les ingénieurs des travaux publics étaient profondémment convaincus que Lisbonne pouvait devenir, grâce au chemin de fer, le port de la Péninsule ibérique et de l'Europe dans le cadre des échanges avec les Amériques et l'Afrique. Ces idées qui associaient le progrès à un retour au passé étaient partagées par la bourgeoisie commerçante et par des étrangers. Elles sont à l'origine de la priorité accordée aux liaisons internationales lors de la construction des premières voies. La ville de Porto, jalouse du rôle attribué à Lisbonne, a aussi exigé une liaison directe avec l'Europe.

Ces objectifs, généralement admis, ont produit dans un contexte de pénurie financière un réseau ferroviaire arborescent. La recherche des courants pré-existants de transport de marchandises et de personnes a produit une accentuation des caractéristiques antérieures du réseau routier à peine atténuée par la recherche des tracés moyens avec un coût de construction faible.

Une carte éditée aux presses nationales représente, dès 1869 , une ligne reliant le Nord au Sud par l'intérieur ${ }^{10}$. Vers 1877 , les ingénieurs des travaux publics et plus 
particulièrement Sousa Brandão, le plus brillant d'entre eux, étaient convaincus de la nécessité de construire cette ligne de façon à relier les deux réseaux de l'Etat, celui de Minho et Douro et celui de Sud et Sud Est. C'est sur cette ligne qu'ils espéraient embrancher le chemin de fer du Tage venu de Madrid. Le premier projet de réseau présenté au Parlement en 1879 par Lourenço de Carvalho, avec de légères modifications, manifestait encore cette volonté.

Cependant, l'Etat n'a pas eu la force de faire construire une telle ligne. Il a dû transiger avec la Compagnie Royale dont l'embranchement de Caceres est devenu liaison internationale (M.P.C.) et qui a construit, après bien des hésitations, la ligne de Beira Baixa s'arrêtant à Guarda. Cette voie ferrée n'assurait pas la liaison entre le Nord, le Centre et le Sud intérieur. La seule liaison Nord/Sud demeura la ligne de Lisbonne à Porto appartenant à la même compagnie.

Loin d'égaliser les conditions de commercialisation des produits, le chemin de fer a accentué les inégalités déjà existantes. L'intérieur en a souffert. Une ville comme Covilhã, qui avait connu une importante croissance industrielle sans le chemin de fer, auquel elle ne fut liée qu'en 1891, n'aura qu'une liaison très indirecte avec ses marchés situés surtout dans le Nord intérieur.

Les intérêts de la Compagnie Royale ont aussi rendu très compliquée la construction d'une voie de chemin de fer desservant à l'ouest les nouveaux producteurs de vins. La compagnie n'acceptait pas la construction d'une voie dont elle n'était pas le concessionnaire, tandis que les députés et les militaires ne voulaient pas que la même compagnie dominât les deux voies pénétrant le système de défense de la capitale. La compagnie a trouvé en Henry Burnai un constructeur factice mais ce n'est qu'après des altérations du tracé et la prise de sa direction par des Portugais que le chemin de fer de l'Ouest a été construit. Ce délai a rendu l'effort trop tardif pour soutenir la viticulture de la région qui a connu le phylloxéra en même temps que l'arrivée du chemin de fer ${ }^{11}$.

L'Etat a aussi conservé le droit d'approuver les tarifs généraux et spéciaux exigeant l'égalité des prix de transport à égalité de conditions. Néanmoins, dans les années 1860, il a perdu le droit de faire baisser les tarifs. En 1858, les ingénieurs des travaux publics avaient fait approuver un tarif dont le prix unitaire diminuait avec l'augmentation de la distance mais ils n'ont pas pu le maintenir. Le contrat avec D. José de Salamanca fut accompagné de l'approbation d'un tarif qui était la traduction du tarif français. Ce tarif maintenait un rapport entre le prix et la distance et il ne contribuait guère à diminuer les handicaps de l'intérieur. Il demeura en vigueur durant toute la période étudiée malgré quelques critiques des agriculteurs et des études des ingénieurs. L'approbation de tarifs spéciaux fit baisser le prix moyen du transport par chemin de fer, mais ces tarifs ne concernaient pas tous les voyageurs ni toutes les marchandises.

L'Etat portugais a fait un effort très important pour soutenir la construction des chemins de fer dans le pays. Il l'a fait dans des circonstances financières qui n'étaient 
pas les meilleures puisque, même après 1853 , il avait beaucoup de mal à faire face aux charges de sa dette. Celle-ci correspondait au départ, pour l'essentiel, au coût de la guerre civile.

Pour soutenir la construction des chemins de fer par des entreprises privées, l'Etat a fait appel à l'émission d'emprunts extérieurs dont le taux d'intérêt très élevé était à l'origine de flux négatifs de capitaux très importants. Les entreprises concessionnaires des chemins de fer obtenaient de la même façon leurs capitaux et leurs commandes de produits industriels étaient effectuées presque entièrement à l'étranger.

Quand l'Etat a construit ses chemins de fer, il a aussi obtenu des capitaux sur le marché intérieur à des taux élevés et on peut affirmer qu'ils concurrençaient l'industrie. Nous savons néanmoins que les banques portugaises achetaient, à la même époque, des fonds espagnols et péruviens.

Malgré tout ce qu'il a dépensé, l'Etat n’a pas réussi à imposer un réseau de chemin de fer totalement conforme aux études de ses ingénieurs. Le réseau dont les premiers objectifs furent internationaux et dont les produits furent souvent insuffisants a accentué les handicaps des régions intérieures.

Il est évident que le Portugal n’a pas dépassé le seuil du développement pendant cette période ; il a cependant connu une croissance considérable. Cette croissance peut être constatée dans l'évolution même des tonnages kilométriques sur voie ferrée qui passent de l'indice 466 en 1877 à 648 en 1892.

C'est cette croissance qui explique que l'Etat portugais soit parvenu à maintenir si longtemps sa politique de travaux publics. Si elles n'ont pas empêché la croissance du déficit, les recettes fiscales de l'Etat se situaient en 1890/91 à l'indice 367 en prenant pour base 100 l'année 1852/53. Le chemin de fer n'en était pas le seul responsable des dépenses : d'autres travaux publics, les dépenses militaires liées à l'expansion coloniale et surtout les charges de la dette publique y étaient pour quelque chose.

Le bilan de cet effort fut considéré comme négatif par une bonne partie des contemporains. A propos des mesures à prendre en 1852 on associait les chemins de fer, l'abondance de capitaux et l'éducation populaire, pour laquelle on dépensa très peu, mais, de cette trilogie, on attendait automatiquement le progrès. 


\section{NOTES}

1 Alexandre, M. Valentin, «Un momento crucial do sub-de-senvolvimento português» dans Ler História n 7 de 1986.

2 Fontvieille, Louis, «Dépenses publiques et problématique de la dévalorisation du capital», A.E.S.C., 1978, pp. 240/253.

3 Rosanvallon, P., La crise de l'État Providence, Paris, 1981, p. 68.

4 Archives Historiques Parlementaires, Lisbonne «Pareceres sobre caminhos de ferro" «relatório da comiss o nomeada a 18 de Julho de 1851 para examinar a proposta Hislop».

5 Données compilées des rapports des ministres des Finances, Travaux Publics et comptes généraux de l'Etat.

6 A.E.F. Paris B31245, Coupure de presse de L'Information du 23 juillet 1912.

7 Archives du Ministère des Travaux Publics, D.G.O.P., vente d'actions.

${ }^{8}$ Larcher, Jaime, $O$ Resgate dos caminhos de ferro de Norte e Leste e a rede do Estado, Lisbonne 1883.

9 Resumos estatisticos dos caminhos de ferro de sul e sueste.

10 Documentos relativos aos caminhos de ferro do sul e sueste e seus prolongamentos, Lisbonne 1869.

11 Pereira, Miriam Halpern, Livre cámbio e desenvolvimento económioo, Lisbonne, 1973. 


\section{Annexe 1}

Dépenses de construction des chemins de fer de l'Etat

\begin{tabular}{|l|r|r|}
\hline Années & Dépenses en milliers de reis & \% du total du pays \\
\hline 1869 & 34171 & 100,00 \\
1870 & 69864 & 100,00 \\
1871 & 136952 & 100,00 \\
1872 & 489650 & 100,00 \\
1873 & 1524863 & 100,00 \\
1874 & 2148810 & 100,00 \\
1875 & 2706829 & 95,95 \\
1876 & 3383290 & 92,74 \\
1877 & 3491816 & 97,95 \\
1878 & 1627867 & 100,00 \\
1879 & 500751 & 73,33 \\
1880 & 122769 & 2,53 \\
1881 & 89826 & 2,31 \\
1882 & 265025 & 17,50 \\
1883 & 40040 & 80,56 \\
1884 & 191152 & 73,76 \\
1885 & 375927 & 15,60 \\
1886 & 588871 & 16,10 \\
1887 & 718112 & 16,78 \\
1888 & 697297 & 11,22 \\
1889 & 307798 & 5,83 \\
1890 & 351164 & 9,34 \\
& & \\
\hline
\end{tabular}


Annexe 2

Chemins de fer de l'Etat ; pourcentage du total du réseau.

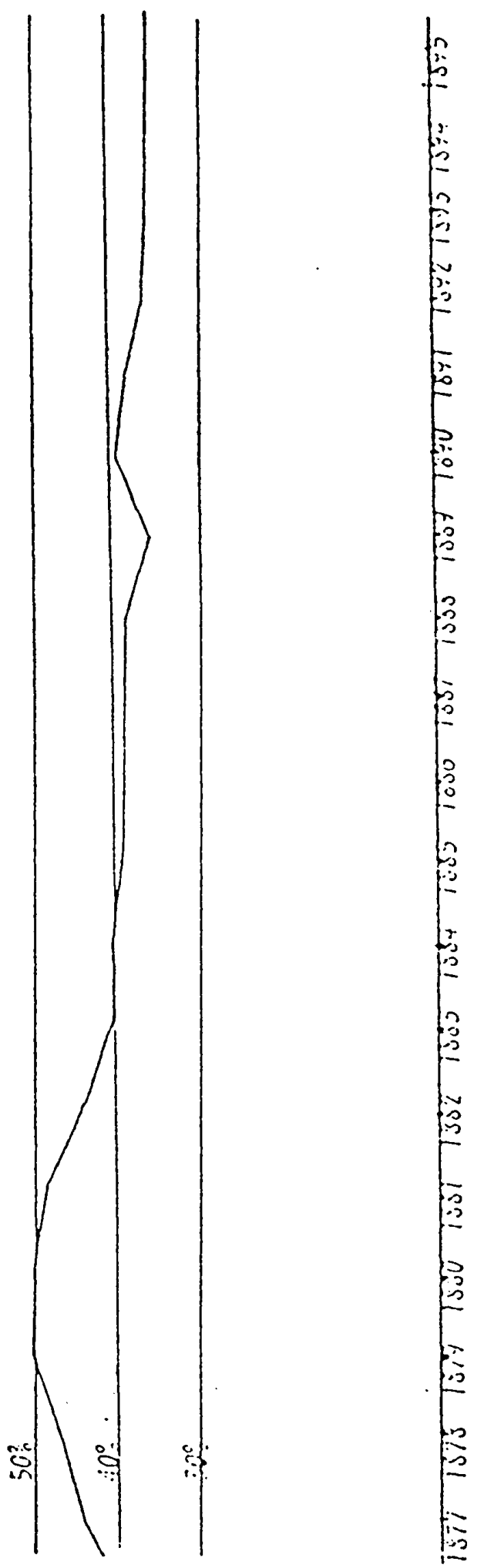


Annere 3

Demande de transports

Transports de marchandises par chemins de fer (petite vitesse-1000 tonnes)

Total du réseau Chemins de fer exploités par des Compagnies privées

Graphique établi d'après Resumos Estatisticos dos caminhos de ferra, A.E.F. Paris B 31246

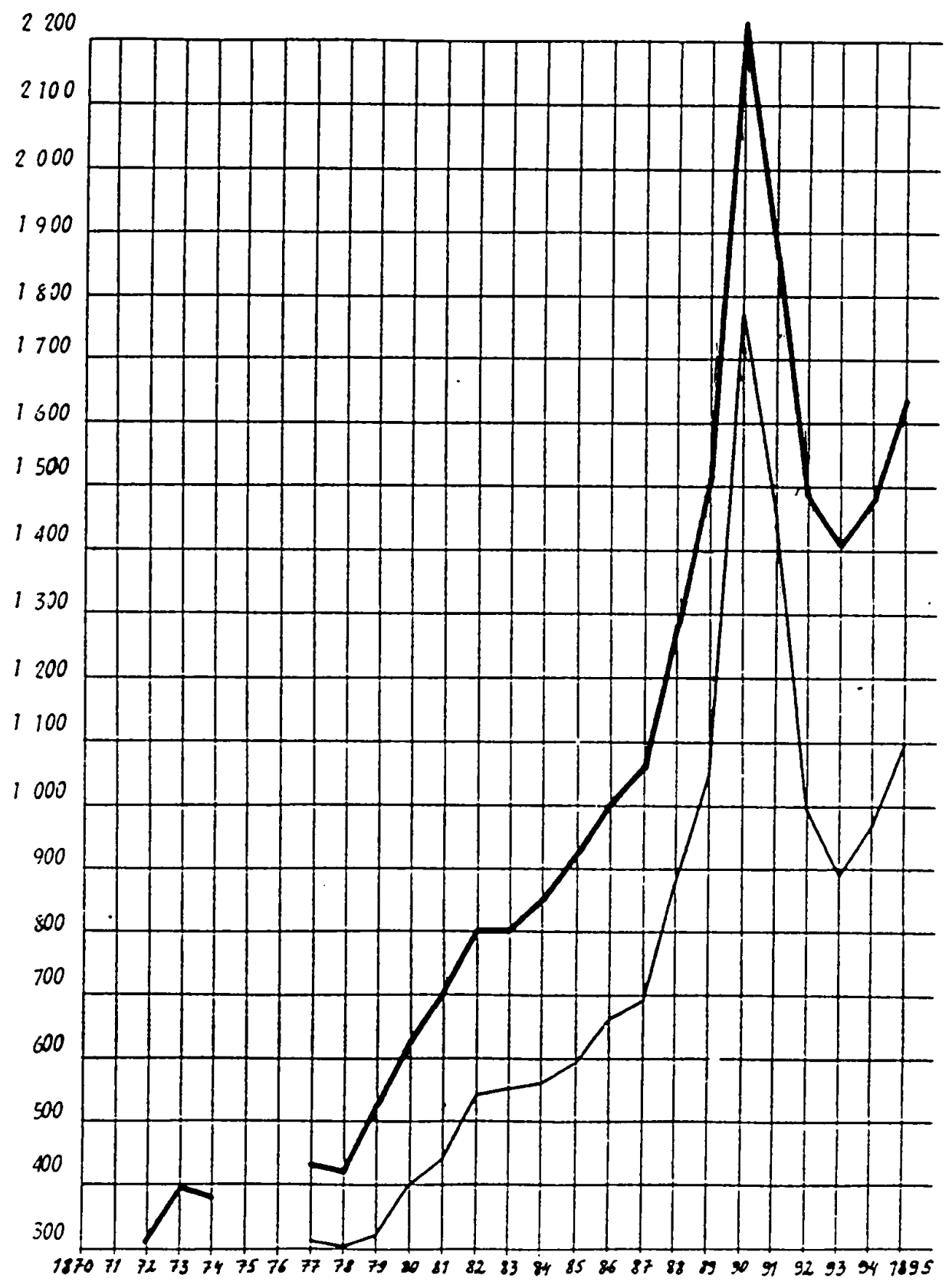




\section{Annexe 4}

\section{Carte $\mathbf{n}^{\circ} 1$}

Chemins de fer ouverts au public jusqu'en 1897

\section{- Compagnie Royale des C.F.P. ---- Chemins de fer de l'Etat \\ -... C.P. Beira Alta Autres compagnies}

Carte établie d'après Legislação sobre caminhos de ferro, vol. I et II, Lisbonne. I.N., 1883 et 1888. Documents relatifs à la combinaison proposée pour l'achèvement de la ligne Vendas Novas à Santt'Anna, Paris Imprimerie de Chaix, 1899.

\section{Carte $n^{\circ} 2$}

Chemins de fer construits et concessions prévues selon le projet de Lourenço de Carvalho - 1879

\section{- Chemins de fer construits $\quad$-.... Concessions prévues}

Carte établie selon Legislação sobre caminhos de ferra, Lisbonne I.N., 1883, p. 391.
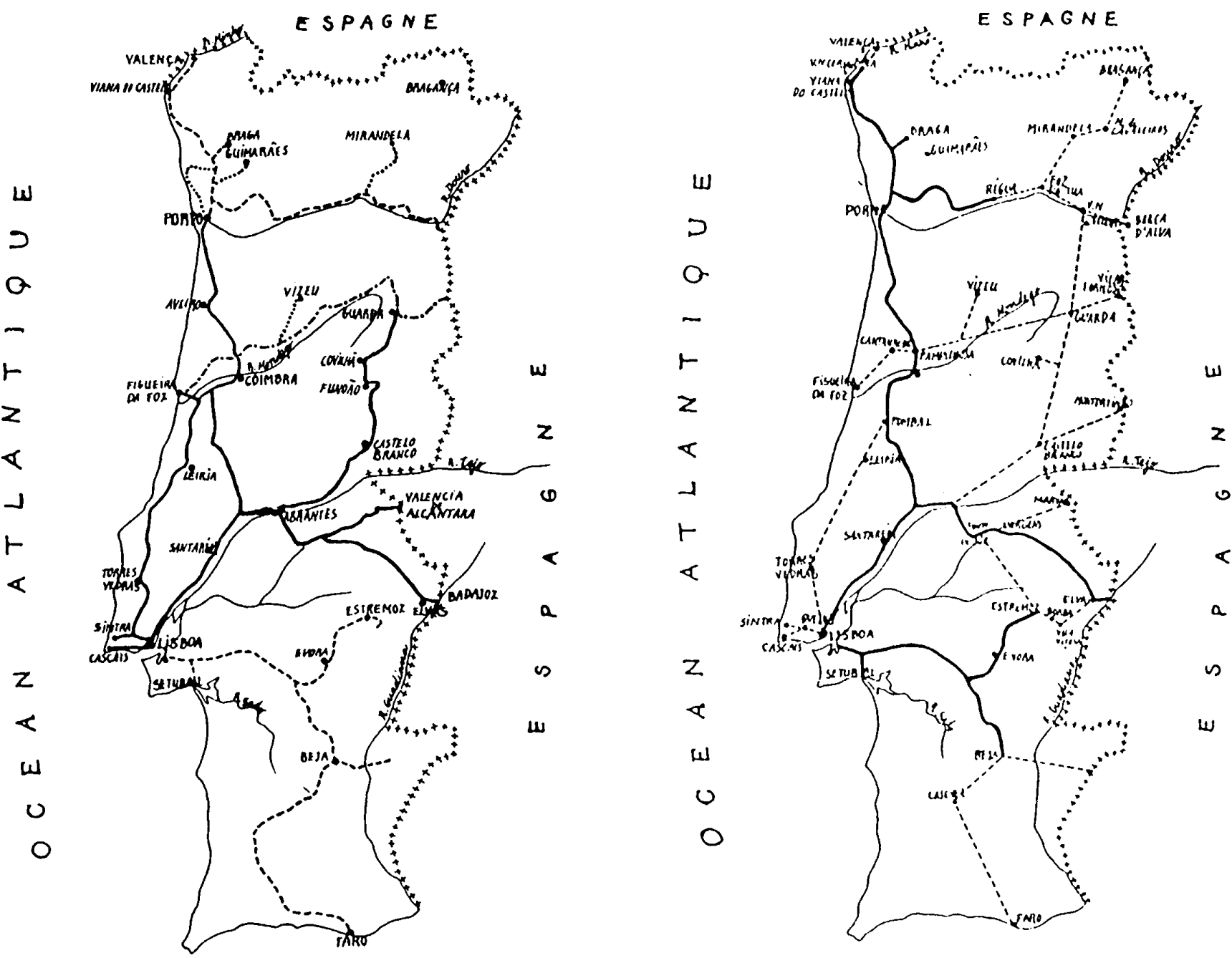